\title{
Author Correction: Crizotinib-induced immunogenic cell death in non-small cell lung cancer
}

\author{
Peng Liu (1) 1,2,3,4,5,6, Liwei Zhao 1,2,3,4,5,6, Jonathan Pol2,3,4,5,6, Sarah Levesque 1,2,3,4,5,6, Adriana Petrazzuolo 1,2,3,4,5,6, \\ Christina Pfirschke ${ }^{7}$, Camilla Engblom ${ }^{7,8}$, Steffen Rickelt (10 9, Takahiro Yamazaki ${ }^{10}$, Kristina Iribarren 2,3,4,5,6, \\ Laura Senovilla 2,3,4,5,6, Lucillia Bezu,2,3,4,5,6,11, Erika Vacchelli2,3,4,5,6, Valentina Sica2,3,4,5,6, Andréa Melis ${ }^{12,13,}$ \\ Tiffany Martin ${ }^{12,13}$, Lin Xia 14,15, Heng Yang 14,15, Qingqing Li ${ }^{14,15}$, Jinfeng Chen ${ }^{14,15}$, Sylvère Durand 2,3,4,5,6, \\ Fanny Aprahamian2,3,4,5,6, Deborah Lefevre 2,3,4,5,6, Sophie Broutin ${ }^{16}$, Angelo Paci ${ }^{16,17}$, Amaury Bongers ${ }^{16}$, \\ Veronique Minard-Colin ${ }^{18}$, Eric Tartour (1) 19,20, Laurence Zitvogel1,15,18,21,22, Lionel Apetoh ${ }^{12,13}$, Yuting Ma (1) 14,15, \\ Mikael J. Pittet ${ }^{7,23}$, Oliver Kepp ${ }^{1,2,3,4,5,6}$ \& Guido Kroemer $2,3,4,5,6,15,24,25$
}

Correction to: Nature Communications https://doi.org/10.1038/s41467-019-09415-3, published online 2 April 2019

The original version of this Article contained an error in the spelling of the author Lin Xia, which was incorrectly given as Xia Lin. This has now been corrected in both the PDF and HTML versions of the Article.

Published online: 17 April 2019

\begin{abstract}
(c) (i)
Open Access This article is licensed under a Creative Commons Attribution 4.0 International License, which permits use, sharing, adaptation, distribution and reproduction in any medium or format, as long as you give appropriate credit to the original author(s) and the source, provide a link to the Creative Commons license, and indicate if changes were made. The images or other third party material in this article are included in the article's Creative Commons license, unless indicated otherwise in a credit line to the material. If material is not included in the article's Creative Commons license and your intended use is not permitted by statutory regulation or exceeds the permitted use, you will need to obtain permission directly from the copyright holder. To view a copy of this license, visit http://creativecommons.org/licenses/by/4.0/.
\end{abstract}

(C) The Author(s) 2019

\footnotetext{
${ }^{1}$ Faculty of Medicine, University of Paris Sud, Kremlin-Bicêtre 94270, France. ${ }^{2}$ Cell Biology and Metabolomics Platforms, Gustave Roussy Cancer Campus, Villejuif 94805, France. ${ }^{3}$ Equipe 11 labellisée Ligue Nationale contre le Cancer, Centre de Recherche des Cordeliers, Paris 75006, France. ${ }^{4}$ Institut National de la Santé et de la Recherche Médicale, UMR1138, Equipe labellisée Ligue Nationale Contre le Cancer, Paris 75006, France. ${ }^{5}$ Université Paris Descartes, Sorbonne Paris Cité, Paris 75006, France. ${ }^{6}$ Université Pierre et Marie Curie, Paris 75006, France. ${ }^{7}$ Center for Systems Biology, Massachusetts General Hospital Research Institute and Harvard Medical School, Boston 02139 MA, USA. ${ }^{8}$ Graduate Program in Immunology, Harvard Medical School, Boston 02238 MA, USA. ${ }^{9}$ Koch Institute for Integrative Cancer Research, Massachusetts Institute of Technology, Cambridge 02139 MA, USA. ${ }^{10}$ Department of Radiation Oncology, Weill Cornell Medical College, New York 14853 NY, USA. ${ }^{11}$ Department of Anaesthesiology, Hôpital Européen Georges Pompidou, Paris 75015, France. ${ }^{12}$ Centre de Recherche INSERM LNC-, UMR1231 Dijon, France. ${ }^{13}$ Department of Medical Oncology, Centre Georges-François Leclerc, Dijon 21000, France. ${ }^{14}$ Center for Systems Medicine, Institute of Basic Medical Sciences, Chinese Academy of Medical Sciences and Peking Union Medical College, Beijing 100730, China. ${ }^{15}$ Suzhou Institute of Systems Medicine, Suzhou 215123 Jiangsu, China. ${ }^{16}$ Department of Pharmacology, Institut Gustave Roussy, Villejuif 94805, France. ${ }^{17}$ School of Pharmacy, Université Paris Sud, Châtenay-Malabry 92 296, France. ${ }^{18}$ Institut de Cancérologie, Gustave Roussy Cancer Campus (GRCC), Villejuif 94805, France. ${ }^{19}$ INSERM U970, Université Paris Descartes Sorbonne Paris-Cité, Paris 75006, France. ${ }^{20}$ Department of Immunology, Hôpital Européen Georges Pompidou, Paris 75015, France. ${ }^{21}$ INSERM U1015, Villejuif 94805, France. ${ }^{22}$ Center of Clinical Investigations CIC1428, Villejuif 94805, France. ${ }^{23}$ Department of Radiology, Massachusetts General Hospital, Boston 02114 MA, USA. ${ }^{24}$ Pôle de Biologie, Hôpital Européen Georges Pompidou, AP-HP, Paris 75015, France. ${ }^{25}$ Department of Women's and Children's Health, Karolinska University Hospital, Stockholm 141 86, Sweden. These authors contributed equally: Peng Liu, Liwei Zhao. These authors jointly supervised this work: Oliver Kepp, Guido Kroemer. Correspondence and requests for materials should be addressed to O.K. (email: captain.olsen@gmail.com) or to G.K. (email: kroemer@orange.fr)
} 